\title{
Low-power, highly reliable dynamic thermal management by exploiting approximate computing
}

\begin{abstract}
With the continuous downscaling of semiconductor processes, the growing power density and thermal issues in multicore processors become more and more challenging, thus reliable dynamic thermal management (DTM) is required to prevent severe challenges in system performance. The accuracy of the thermal profile, delivered to the DTM manager, plays a critical role in the efficiency and reliability of DTM, different sources of noise and variations in deep submicron (DSM) technologies severely affecting the thermal data that can lead to significant degradation of DTM performance. In this article, we propose a novel fault-tolerance scheme exploiting approximate computing to mitigate the DSM effects on DTM efficiency. Approximate computing in hardware design can lead to significant gains in energy efficiency, area, and performance. To exploit this opportunity, there is a need for design abstractions that can systematically incorporate approximation in hardware design which is the main contribution of our work. Our proposed scheme achieves $11.20 \%$ lower power consumption, $6.59 \%$ smaller area, and $12 \%$ reduction in the number of wires, while increasing DTM efficiency by $5.24 \%$.
\end{abstract}

Keyword: Thermal management; Reliability; Approximate computing; Temperature sensors; Thermal noise 